\title{
Symbolic Computation and Construction of Soliton-like Solutions of some Nonlinear Evolution Equations
}

\author{
Heng-Nong Xuan and Biao $\mathrm{Li}^{\mathrm{a}}$ \\ Department of Computer Science and Technology, Nanjing University of Economics, \\ Nanjing 210003, People's Republic of China \\ a Department of Applied Mathematics, Dalian University of Technology, \\ Dalian 116024, People's Republic of China \\ Reprint requests to Dr. B. Li; E-mail: libiao@dlut.edu.cn
}

Z. Naturforsch. 58a, 167 - 175 (2003); received December 11, 2002

Based on the computerized symbolic computation system Maple and a Riccati equation, a new generalized Riccati equation expansion method for constructing non-travelling waves and coefficient functions' soliton-like solutions of nonlinear evolution equations (NEEs) is presented by a general ansatz. Compared with most of the existing tanh methods, namely the extended tanh-function method, the modified extended tanh-function method and the generalized hyperbolic-function method, the proposed method is more powerful. By use of the method one can not only successfully recover the previously known formal solutions but also construct new and more general formal solutions for some NEEs. The cylindrical Korteweg-de Vries (CkdV) equation, a Potential Kadomstev-Petviashvili (PKP) equation, the two-dimensional KdV-Burgers equation are chosen to illustrate our method such that rich new families of exact solutions, including the non-travelling wave soliton-like solutions, singular soliton-like solutions, periodic form solutions are obtained. When taking arbitrary functions of the solutions as some special constants, the known travelling wave solutions of the PKP equation, two-dimensional KdV-Burgers equation can be recovered.
Key words: Generalized Riccati Equation Expansion Method; CKdV Equation; PKP Equation;
Two-dimensional KdV-Burgers Equation; Soliton-like Solutions; Soliton;
Symbolic Computation.

\section{Introduction}

In recent years, directly searching for exact solutions of nonlinear evolution equations (NEEs) has become more and more attractive, on one hand due to their occurrence in many fields of science, physics as well as chemistry and biology, and the interesting features and rich variety of their solutions, on the other hand due to the availability of computer systems like Maple or Mathematica, which allow to perform complicated and tedious algebraic calculations and differential calculations on a computer such that one may find new exact solutions of NEEs. Many powerful methods have been developed, such as, the inverse scattering method, Bäcklund transformation, Darboux transformation, Hirota method [1-5], tanh method [6-10], extended tanh-function method [11, 12], modified extended tanh-function method [17], generalized hyperbolic-function method [18,19], etc. Particularly, various ansätze have been proposed in order to obtain new forms of solutions.
The tanh method [6-10] is a most effectively straightforward method to construct exact travelling wave solutions of NEEs. Recently, Fan [11,12] has proposed an extended tanh-function method. More recently, Fan et al. [13], Yan [14-16] and Li et al. [20-23] further developed this idea and made it much more lucid and straightforward for a class of NEEs. Most recently, Elwakil et al. [17] modified the extended tanh-function method and obtain some new formal exact solutions. To obtain the solitonlike solutions for NEEs, Gao and Tian $[18,19]$ presented a generalized hyperbolic-function method. As we known, when applying a directed method, the choice of an appropriate ansatz is of great importance. In this paper, based on [6-23], by introducing a new more general ansatz we present a generalized Riccati equation expansion method. Then we choose the cylindrical Korteweg-de Vries equation, the Potential Kadomstev-Petviashvili equation and the twodimensional $\mathrm{KdV}$-Burgers equation to illustrate our algorithm and obtain rich new families of exact solu- 
tions, including non-travelling wave soliton-like solutions, singular soliton-like solutions, and periodic form solutions.

\section{Generalized Riccati Equation Expansion Method}

We establish the generalized Riccati equation expansion method as follows:

Step 1. For a given NEE with a physical field $u(x, y, t)$

$$
H\left(u, u_{t}, u_{x}, u_{y}, u_{x x}, u_{x t}, u_{x y}, u_{y t}, \cdots\right)=0
$$

we express the solutions of (2.1) by the new more general ansatz

$$
\begin{gathered}
u(x, y, t)=a_{0}+\sum_{i=1}^{m}\left[a_{i} \phi^{i}(\xi)+b_{i} \phi^{i-1}(\xi) \sqrt{R+\phi^{2}(\xi)}\right. \\
\left.+k_{i} \phi^{-i}(\xi)\right]
\end{gathered}
$$

where $m$ is an integer to be determined by balancing the highest order derivative terms with the nonlinear terms in (2.1). $R$ is a real constant. $a_{0}=a_{0}, a_{i}, b_{i}, k_{i}$, $(i=1, \cdots, m)$, and $\xi$ are unknown functions of $x, y$ and $t$, and $\phi(\xi)$ satisfies

$$
\frac{\mathrm{d} \phi(\xi)}{\mathrm{d} \xi}=R+\phi^{2}(\xi) \text {. }
$$

Step 2. Substituting (2.2) along with (2.3) into (2.1), multiplying with the most simple common denominator in the obtained system, setting to zero the coefficients of $\phi^{j}(\xi)\left(\sqrt{R+\phi^{2}(\xi)}\right)^{n}(j=0,1, \cdots$; $n=0,1$ ) (Note: $\phi^{j}(\xi)$ denotes the $j$-th power of $\phi(\xi)$ and $\left(\sqrt{R+\phi^{2}(\xi)}\right)^{n}$ denotes the $n$-th power of $\left.\sqrt{R+\phi^{2}(\xi)}\right)$, we obtain a set of over-determined partial differential equations (PDEs) with respect to unknown functions $a_{0}, a_{i}, b_{i}, k_{i}(i=1, \cdots, m)$ and $\xi$.

Step 3. Solving the over-determined PDEs by use of the PDEtools package of Maple, we end up with explicit expressions for $a_{0}, a_{i}, b_{i}, k_{i}(i=1, \cdots, m)$ and $\xi$ or the constrains among them.

Step 4. It is well-known that the general solutions of Riccati equation (2.3) are

1. When $R<0$,

$$
\phi(\xi)=\left\{\begin{array}{c}
-\sqrt{-R} \tanh (\sqrt{-R} \xi) \\
-\sqrt{-R} \operatorname{coth}(\sqrt{-R} \xi)
\end{array},\right.
$$

2. When $R=0$,

$$
\phi(\xi)=-\frac{1}{\xi},
$$

3. When $R>0$,

$$
\phi(\xi)=\left\{\begin{array}{l}
\sqrt{R} \tan (\sqrt{R} \xi) \\
-\sqrt{R} \cot (\sqrt{R} \xi)
\end{array} .\right.
$$

Thus, according to (2.2), (2.4) - (2.6) and the conclusions in Step 3, we obtain the soliton-like solutions, singular soliton-like solutions and periodic form solutions of (2.1).

Remark 1. The method proposed here is more general than the generalized hyperbolic-function method $[18,19]$, tanh method [6-10], extended tanh-function method [11 - 16], and modified extended tanh-function method [17]. Firstly, compared with the tanh method, extended tanh-function, as well as the modified extended tanh-function method, the restriction on $\xi(x, y, t)$ as merely a linear function of $x, y, t$ and the restriction on the coefficients $a_{0}, a_{i}, b_{i}, k_{i}(i=1, \cdots, m)$ as constants is removed. Secondly, compared with the generalized hyperbolic-function method, we can not only recover the exact soliton-like solutions for given NEEs, but also with no extra effect, find new and more general solutions, such as singular soliton-like solutions and periodic solutions. More importantly, we add terms $k_{i} \phi^{-i}(\xi)$ in the ansatz (2.2), so more types of solutions can be expected for some equations.

Remark 2. For the generalization of the ansatz, naturally more complicated computations are expected than before. Even if computer symbolic systems like Maple or Mathematica allow us to perform the complicated and tedious algebraic calculations and differential calculations on a computer, in general it is very difficult, sometime impossible, to solve the set of overdetermined PDEs in Step 2. As the calculation goes on, in order to drastically simplify the work or make the work feasible, we often choose special function forms for $a_{0}, a_{i}, b_{i}, k_{i}(i=1, \cdots, m)$ and $\xi$, on a trial-anderror basis.

\section{Applications of the Method}

\section{Example 1. The Cylindrical Korteweg-de Vries Equation}

Consider the cylindrical Korteweg-de Vries equation $[2,3]$ 
H.-N. Xuan and B. Li · Soliton-like solutions of Some Nonlinear Evolution Equations

$$
u_{t}+6 u u_{x}+u_{x x x}+\frac{1}{2 t} u=0 .
$$

In order to facilitate the computation, we change (3.1) into the following form

$$
2 t\left(u_{t}+6 u u_{x}+u_{x x x}\right)+u=0 .
$$

By balancing the highest order contributions from both the linear and nonlinear terms in (3.2) or (3.1), we obtain $m=2$ in (2.2). Therefore we assume for the solutions of (3.1) the special form

$$
u(x, t)=x a_{0}(t)+a_{1}(t) \phi(\xi)+a_{2}(t) \phi(\xi)^{2},
$$

where $\xi=x p(t)+q(t)$ and $\phi(\xi)$ satisfy (2.3).

Substituting (3.3) and (2.3) into (3.2) and collecting the coefficients of the monomials of $\phi(\xi)$ and $x$ (Note that $a_{0}, a_{1}, a_{2}, p$ and $q$ are independent of $x$ ), then setting each coefficients to zero, we obtain

$$
\begin{gathered}
24 t a_{2} p\left(2 p^{2}+a_{2}\right)=0 \\
12 t a_{1} p\left(p^{2}+3 a_{2}\right)=0 \\
2 t R a_{1}\left(q_{t}+2 R p^{3}\right)=0 \\
12 t a_{0} p a_{1} R+12 t a_{0}^{2}+a_{0}+2 t a_{0 t}+2 t a_{1} R p_{t}=0 \\
2 t a_{1 t}+4 t a_{2} R q_{t}+12 t a_{1} a_{0}+12 t a_{1}^{2} p R \\
+32 t p^{3} a_{2} R^{2}+a_{1}=0 \\
4 t R a_{2}\left(p_{t}+6 a_{0} p\right)=0 \\
36 t a_{1} p a_{2} R+16 t p^{3} a_{1} R+2 t a_{1} q_{t} \\
+12 t a_{2} a_{0}+a_{2}+2 t a_{2 t}=0 \\
2 t a_{1}\left(p_{t}+6 a_{0} p\right)=0 \\
4 t\left(6 a_{2}^{2} p R+3 a_{1}^{2} p+20 p^{3} a_{2} R+a_{2} q_{t}\right)=0 \\
4 t a_{2}\left(p_{t}+6 a_{0} p\right)=0
\end{gathered}
$$

where $p_{t}=\partial p / \partial t, a_{i t}=\partial a_{i} / \partial t(i=0,1,2)$.

Using the powerful PDEtools package of Maple in solving the set of partial differential equations (3.4)(3.13), we obtain the following non- trivial (i.e., $p \neq 0$ ) solutions:

$$
a_{1}=0, \quad a_{0}=\frac{1}{12 t}, \quad a_{2}=\frac{C_{1}}{t},
$$

$$
q=-4 R C_{1}^{2} \sqrt{-\frac{2}{C_{1} t}}+C_{2}, \quad p=-\frac{\sqrt{-2 C_{1} t}}{2 t}
$$

and

$$
\begin{aligned}
& a_{1}=0, \quad a_{0}=\frac{1}{12 t}, \quad a_{2}=\frac{C_{1}}{t} \\
& q=4 R C_{1}^{2} \sqrt{-\frac{2}{C_{1} t}}+C_{2}, \quad p=\frac{\sqrt{-2 C_{1} t}}{2 t},
\end{aligned}
$$

where $C_{1}, C_{2}$ are arbitrary constants.

Thus we can obtain the following solutions of (3.1):

$$
\begin{aligned}
u_{11}=\frac{x}{12 t}-\frac{C_{1} R}{t} \tanh ^{2}[ & \pm \frac{x \sqrt{-2 C_{1} t}}{2 t} \\
& \left. \pm 4 R C_{1}^{2} \sqrt{-\frac{2}{C_{1} t}}+C_{2}\right], R<0
\end{aligned}
$$

$u_{12}=\frac{x}{12 t}-\frac{C_{1} R}{t} \operatorname{coth}^{2}\left[ \pm \frac{x \sqrt{-2 C_{1} t}}{2 t}\right.$

$$
\left. \pm 4 R C_{1}^{2} \sqrt{-\frac{2}{C_{1} t}}+C_{2}\right], R<0,
$$

$u_{13}=\frac{x}{12 t}+\frac{C_{1} R}{t} \tan ^{2}\left[ \pm \frac{x \sqrt{-2 C_{1} t}}{2 t}\right.$

$$
\left. \pm 4 R C_{1}^{2} \sqrt{-\frac{2}{C_{1} t}}+C_{2}\right], R>0
$$

$$
\begin{aligned}
u_{14}=\frac{x}{12 t}+\frac{C_{1} R}{t} \cot ^{2}[ & \pm \frac{x \sqrt{-2 C_{1} t}}{2 t} \\
& \left. \pm 4 R C_{1}^{2} \sqrt{-\frac{2}{C_{1} t}}+C_{2}\right], R>0 .
\end{aligned}
$$

Remark 3. It is necessary to point out that, when setting

$$
\begin{aligned}
u(x, t)= & x a_{0}(t)+a_{1}(t) \phi(\xi)+a_{2}(t) \phi(\xi)^{2} \\
& +b_{1}(t) \sqrt{R+\phi(w)^{2}}+b_{2}(t) \phi(\xi) \sqrt{R+\phi(\xi)^{2}} \\
& +k_{1}(t) / \phi(\xi)+k_{2}(t) / \phi(\xi)^{2},
\end{aligned}
$$

the over-determined partial differential equations can not be solved by use of the PDEtools package of Maple. Therefore we consider only the solutions of (3.1) in the form (3.2).

Example 2. Potential Kadomstev-Petviashvili (PKP) Equation

Let us consider a $(2+1)$-dimensional generalization of the Korteweg de Vries equation of the form 


$$
u_{t}+\frac{3}{4} u_{x}^{2}+\frac{1}{4} \int^{x} u_{y y} \mathrm{~d} x^{\prime}=0
$$

which describes the dynamics of 2-dimensional, small, but finite amplitude waves and solitons in a variety of media, for example, in plasma physics, hydrodynamics and solid-state physics. Equation (3.21) is also derived in various physical contexts assuming that the wave is moving along $x$ and all changes in $y$ are slower than in the direction of motion [2,3].

For our purpose it is convenient to consider (3.21) in the form

$$
4 u_{x t}+6 u_{x} u_{x x}+u_{x x x x}+3 u_{y y}=0
$$

which is often called PKP equation. Senthivelan [24] considered the solitary wave solutions.

Proceeding as before, we assume that the solutions of (3.22) have in the following three special forms:

$$
u_{1}(x, y, t)=a_{0}+a_{1} \phi(\xi)+b_{1} \sqrt{R+\phi(\xi)^{2}}+\frac{k_{1}}{\phi(\xi)},
$$

$u_{2}(x, y, t)=x a_{0}+a_{1} \phi(\xi)+b_{1} \sqrt{R+\phi(\xi)^{2}}+\frac{k_{1}}{\phi(\xi)}$,

$u_{3}(x, y, t)=x^{2} a_{0}+a_{1} \phi(\xi)+b_{1} \sqrt{R+\phi(\xi)^{2}}+\frac{k_{1}}{\phi(\xi)}$

where $a_{0}=a_{0}(t), a_{1}=a_{1}(y, t), b_{1}=b_{1}(y, t), k_{1}=$ $k_{1}(y, t), \xi=x p(t)+q(y, t)$.
Substituting (3.23) $-(3.25)$, respectively, along with (2.3) into (3.22), multiplying by $\phi(\xi)^{5} \sqrt{R+\phi(\xi)^{2}}$ in the obtained system, collecting coefficients of the monomials of $\phi(\xi), \sqrt{R+\phi(\xi)^{2}}$ and $x$ (Notice that $a_{0}$, $a_{1}, b_{1}, k_{1}, p$ and $q$ are independent of $x$ ), then setting each coefficient to zero, we obtain three sets which are respectively composed of 23,24 and 25 partial differential equations with respect to the unknown functions $a_{0}, a_{1}, b_{1}, k_{1}, p$ and $q$. For simplicity, we do not list them. Solving these three sets, using Maple, we obtain the following results $(3.26)-(3.27)$.

Note: In the rest of this paper $F_{1}(t)$ and $F_{2}(t)$ denote arbitrary functions with regard to $t$ and $C_{i}(i=1, \cdots, 8)$ are arbitrary constants.

Case A:

$$
\begin{gathered}
k_{1}=0, b_{1}=C_{1}, a_{0}=F_{3}(t) y+F_{4}(t), a_{1}=C_{1}, p=-C_{1}, \\
q=\frac{1}{4 C_{1}}\left(-t C_{1}^{4} R+\left(4 C_{3} y+4 C_{2}\right) C_{1}+3 t C_{3}^{2}\right) ; \\
k_{1}=0, b_{1}=C_{1}, a 1=-C_{1}, p=C_{1}, \\
q=\frac{1}{4 C_{1}}\left(t C_{1}^{4} R+\left(4 C_{3} y+4 C_{2}\right) C_{1}-3 t C_{3}^{2}\right), \\
a_{0}=F_{1}(t) y+F_{2}(t) ;
\end{gathered}
$$

$$
\begin{aligned}
& a_{0}=F_{1}(t) y+F_{2}(t), b_{1}=0, k_{1}=0, a_{1}=C_{1}, p=-\frac{1}{2} C_{1}, \\
& q=\frac{1}{8 C_{1}}\left(-t C_{1}^{4} R+\left(8 C_{3} y+8 C_{2}\right) C_{1}+12 t C_{3}^{2}\right) ;
\end{aligned}
$$

$$
q=\frac{1}{2 C_{1} R^{2}}\left(t C_{1}^{4}+2 R^{2}\left(C_{3} y+C_{2}\right) C_{1}-3 t R^{3} C_{3}^{2}\right), k_{1}=C_{1},
$$

$a_{0}=F_{1}(t) y+F_{2}(t), b_{1}=0, p=\frac{C_{1}}{2 R}, a_{1}=-\frac{C_{1}}{R}$.

Case B:

$$
\begin{aligned}
& b_{1}=C_{1}, a_{1}=C_{1}, k_{1}=0, a_{0}=C_{4} y+C_{5}, p=-C_{1}, q=\frac{1}{48 C_{1}^{2} C_{4}} \cdot \\
& \quad \cdot\left\{27 C_{1}^{3} C_{4}^{3} t^{3}+\left(72 C_{1}^{3} y t+54 C_{1}^{2} t^{2} C_{7}\right) C_{4}^{2}+\left[-12 C_{1}^{5} R t+72 C_{1}^{3} C_{5} t+\left(48 C_{6}+48 C_{7} y\right) C_{1}^{2}+36 C_{1} t C_{7}^{2}\right] C_{4}+8 C_{7}^{3}\right\} \\
& b_{1}=C_{1}, a_{1}=-C_{1}, k_{1}=0, a_{0}=C_{2} y+C^{3}, p=C_{1}, \\
& q=\frac{1}{48 C_{1}^{2} C_{2}}\left\{12 C_{1}^{5} R t_{C} 2-72\left[\frac{3}{8} C_{2}^{2} t^{2}+C_{2} y+C_{3}\right] C_{2} t C_{1}^{3}+48\left[\frac{9}{8} C_{7} t^{2} C_{2}+C_{6}+C_{7} y\right] C_{2} C_{1}^{2}-36 C_{2} C_{1} t C_{7}^{2}+8 C_{7}^{3}\right\} ; \\
& p=-\frac{1}{2} C_{1}, a_{1}=C_{1}, k_{1}=0, b_{1}=0, a_{0}=C_{2} y+C_{3},
\end{aligned}
$$




$$
\begin{aligned}
& q=\frac{1}{96 C_{1}^{2} C_{2}}\left\{-12 C_{1}^{5} R t C_{2}+72\left[\frac{3}{8} C_{2}^{2} t^{2}+C_{2} y+C_{3}\right] C_{2} t C_{1}^{3}+96 C_{2}\left[\frac{9}{8} t^{2} C_{5} C_{2}+C_{4}+y C_{5}\right] C_{1}^{2}+144 C_{2} C_{1} t C_{5}^{2}+64 C_{5}^{3}\right\} ; \\
& k_{1}=C_{1}, b_{1}=0, a_{0}=C_{2} y+C_{3}, a 1=-\frac{C_{1}}{R}, p=\frac{C_{1}}{2 R}, q=\frac{1}{96 R^{2} C_{1}^{2} C_{2}} . \\
& \cdot\left\{48 C_{1}^{5} t C_{2}-72 R t C_{2}\left[3 / 8 C_{2}^{2} t^{2}+C_{2} y+C_{3}\right] C_{1}^{3}+96\left[\frac{9}{8} t^{2} C_{5} C_{2}+C_{4}+y C_{5}\right] R^{2} C_{2} C_{1}^{2}-144 C_{1} C_{2} t C_{5}^{2} R^{3}+64 C_{5}^{3} R^{4}\right\} .
\end{aligned}
$$

Case C:

$$
\begin{aligned}
& b_{1}=0, a_{1}=\frac{1}{\left(C_{1} t+C_{2}\right) R}, p=-\frac{1}{2\left(C_{1} t+C_{2}\right) R}, k_{1}=-\frac{1}{C_{1} t+C_{2}}, a_{0}=\frac{C_{1}}{3\left(C_{1} t+C_{2}\right)} \\
& q=\frac{1}{\left(C_{1} t+C_{2}\right)^{2} R^{2}}\left\{-\frac{1}{3} y \sqrt{R\left[-\frac{4 t^{3} C_{1}^{3} C_{4} R^{2}}{\left(t+\frac{C_{2}}{C_{1}}\right)^{3}}-\frac{4 C_{4} R^{2} C_{2}^{3}}{\left(t+\frac{C_{2}}{C_{1}}\right)^{3}}-\frac{12 t^{2} C_{1}^{2} C_{4} R^{2} C_{2}}{\left(t+\frac{C_{2}}{C_{1}}\right)^{3}}-\frac{12 t C_{1} C_{4} R^{2} C_{2}^{2}}{\left(t+\frac{C_{2}}{C_{1}}\right)^{3}}+1\right]} \sqrt{3}\right. \\
& \left.+R\left[R\left(C_{4}+t^{2} C_{3}\right) C_{1}^{2}+\left(2 R C_{3} t C_{2}-\frac{1}{3} y^{2}\right) C_{1}+R C_{2}^{2} C_{3}\right]\right\} ; \\
& k_{1}=0, b_{1}=0, a_{1}=-\frac{1}{C_{1} t+C_{2}}, p=\frac{1}{\left.2 C_{1} t+2 C_{2}\right)}, a_{0}=\frac{C_{1}}{3\left(C_{1} t+C_{2}\right)}, \\
& q=\left\{-\frac{1}{6} y \sqrt{3 R+\frac{48 t^{3} C_{1}^{3} C_{4}}{\left(t+\frac{C_{2}}{C_{1}}\right)^{3}}+\frac{144 t C_{1} C_{4} C_{2}^{2}}{\left(t+\frac{C_{2}}{C_{1}}\right)^{3}}+\frac{144 t^{2} C_{1}^{2} C_{4} C_{2}}{\left(t+\frac{C_{2}}{C_{1}}\right)^{3}}+\frac{48 C_{4} C_{2}^{3}}{\left(t+\frac{C_{2}}{C_{1}}\right)^{3}}}+\left(C_{4}+t^{2} C_{3}\right) C_{1}^{2}\right. \\
& \left.+\left(2 C_{3} C_{2} t+\frac{1}{3} y^{2}\right) C_{1}+C_{3} C_{2}^{2}\right\} \frac{1}{\left(C_{1} t+C_{2}\right)^{2}} \\
& k_{1}=0, a_{0}=\frac{C_{1}}{3\left(C_{1} t+C_{2}\right)}, b_{1}=-\frac{1}{C_{1} t+C_{2}}, a_{1}=\frac{1}{C_{1} t+C_{2}}, p=-\frac{1}{C_{1} t+C_{2}}, \\
& q=\left\{-\frac{1}{3} y \sqrt{-\frac{24 C_{4} C_{2}^{3}}{\left(t+\frac{C_{2}}{C_{1}}\right)^{3}}+3 R-\frac{72 t^{2} C_{1}^{2} C_{4} C_{2}}{\left(t+\frac{C_{2}}{C_{1}}\right)^{3}}-\frac{24 t^{3} C_{1}^{3} C_{4}}{\left(t+\frac{C_{2}}{C_{1}}\right)^{3}}-\frac{72 t C_{1} C_{4} C_{2}^{2}}{\left(t+\frac{C_{2}}{C_{1}}\right)^{3}}}+\left(C_{4}+t^{2} C_{3}\right) C_{1}^{2}\right. \\
& \left.+\left(2 C_{3} C_{2} t-\frac{2}{3} y^{2}\right) C_{1}+C_{3} C_{2}^{2}\right\} \frac{1}{\left(C_{1} t+C_{2}\right)^{2}} \\
& k_{1}=0, a_{1}=-\frac{1}{C_{1} t+C_{2}}, a_{0}=\frac{C_{1}}{3\left(C_{1} t+C_{2}\right)}, b_{1}=-\frac{1}{C_{1} t+C_{2}}, p=\frac{1}{C_{1} t+C_{2}}, \\
& q=\left\{\frac{1}{3} y \sqrt{\frac{72 t C_{1} C_{4} C_{2}^{2}}{\left(t+\frac{C_{2}}{C_{1}}\right)^{3}}+\frac{72 t^{2} C_{1}^{2} C_{4} C_{2}}{\left(t+\frac{C_{2}}{C_{1}}\right)^{3}}+3 R+\frac{24 t^{3} C_{1}^{3} C_{4}}{\left(t+\frac{C_{2}}{C_{1}}\right)^{3}}+\frac{24 C_{4} C_{2}^{3}}{\left(t+\frac{C_{2}}{C_{1}}\right)^{3}}}\right. \\
& \left.+\left(C_{4}+t^{2} C_{3}\right) C_{1}^{2}+\left(2 C_{3} C_{2} t+\frac{2}{3} y^{2}\right) C_{1}+C_{3} C_{2}^{2}\right\} \frac{1}{\left(C_{1} t+C_{2}\right)^{2}} \text {. }
\end{aligned}
$$


From (2.4), (2.6) and A) (3.23),(3.26)-(3.29); B) (3.24), (3.30)-(3.33); C) (3.25), (3.34)-(3.37) respectively, we can obtain corresponding solutions of (3.21). For simplicity, we do not list them.

\section{Remark 3.}

1) When setting $a_{0}=a_{0}(y, t)$ or when the coefficient of $a_{0}$ are equal to $f(x)$ in $(3.23)-(3.25)$, solutions of the corresponding over-determined PDEs can not be obtained by Maple.

2) When setting the coefficient of $a_{0}$ equal to $x^{4}$ or $x^{5}$ in (3.23)- (3.25), from the corresponding overdetermined PDEs, only the trivial solutions with $a_{0}=0$ can be obtained. Therefore we only consider three cases in this paper.

3) When setting $F_{1}(t)=0, F_{2}(t)=a_{0}, C_{1}=-2 k \alpha$, $C_{2}=0$ and $C_{3}=k \beta$ in (3.28), the solutions obtained from (2.4), (3.23), (3.28) are the same as solutions in [24].

Example 3. The Two-dimensional KdV-Burgers Equation

The two-dimensional KdV-Burgers equation [8, 9 , $13,17,25,26]$ reads

$$
\left(u_{t}+u u_{x}-\alpha u_{x x}+\beta u_{x x x}\right)_{x}+\gamma u_{y y}=0 .
$$

Proceeding as before, we assume that the solutions of (3.38) have the form

$$
\begin{aligned}
u(x, y, t)= & a_{0}+a_{1} \phi(\xi)+a_{2} \phi(\xi)^{2} \\
& +\left[b_{1}+b_{2} \phi(\xi)\right] \sqrt{R+\phi^{2}(\xi)} \\
& +\frac{k_{1}}{\phi(\xi)}+\frac{k_{2}}{\phi^{2}(\xi)},
\end{aligned}
$$

where $a_{0}, a_{1}, a_{2}, b_{1}, b_{2}, k_{1}$ and $k_{2}$ are functions of $y$ and $t, \xi=x p(y, t)+q(y, t)$, all functions are differentiable and $\phi(\xi)$ satisfies $(2.3)$.

Substituting (3.38b) along with (2.3) into (3.38a), multiplying $\phi(\xi)^{6} \sqrt{R+\phi(\xi)^{2}}$ in the obtained system, collecting the coefficients of the monomials of $\phi(\xi)$, $\sqrt{R+\phi(\xi)^{2}}$ and $x$, then setting each coefficient to zero, we can obtain a set which is composed of 55 partial differential equations with respect to the unknown functions $a_{0}, a_{1}, a_{2}, b_{1}, b_{2}, k_{1}, k_{2}, p$ and $q$. For simplify, we do not list this set. Solving the set using Maple, we can obtain the following non-trivial (i.e., $p \neq 0$ ) solutions.
Case 1.

$$
\begin{gathered}
p=\frac{\alpha}{10 \sqrt{-R} \beta}, a_{0}=\frac{2500 \beta^{3} R \gamma F_{1}(t)^{2}+3 \alpha^{4}-25 \alpha^{2} \beta}{25 \alpha^{2} \beta}, \\
q=F_{1}(t) y+F_{2}(t), b_{1}=b_{2}=a_{1}=a_{2}=0 \\
k_{2}=\frac{3 R \alpha^{2}}{25 \beta}, k_{1}=\frac{6 \alpha^{2} R}{25 \sqrt{-R} \beta} .
\end{gathered}
$$

Case 2.

$$
\begin{aligned}
& p=-\frac{\alpha}{10 \sqrt{-R} \beta}, b_{1}=b_{2}=a_{1}=a_{2}=0, \\
& k_{2}=\frac{3 R \alpha^{2}}{25 \beta}, k_{1}=-\frac{6 R \alpha^{2}}{25 \sqrt{-R} \beta}, \\
& a_{0}=\frac{2500 \beta^{3} R \gamma F_{1}(t)^{2}+3 \alpha^{4}-25 \alpha^{2} \beta}{25 \alpha^{2} \beta}, \\
& q=F_{1}(t) y+F_{2}(t) .
\end{aligned}
$$

Case 3.

$$
\begin{gathered}
p=\frac{\alpha}{10 \sqrt{-R} \beta}, a_{0}=\frac{2500 \beta^{3} R \gamma F_{1}(t)^{2}+3 \alpha^{4}-25 \alpha^{2} \beta}{25 \alpha^{2} \beta}, \\
q=F_{1}(t) y+F_{2}(t), k_{1}=k_{2}=b_{1}=b_{2}=0, \\
a_{2}=\frac{3 \alpha^{2}}{25 R \beta}, a_{1}=-\frac{6 \alpha^{2}}{25 \sqrt{-R} \beta} .
\end{gathered}
$$

Case 4.

$$
\begin{gathered}
p=-\frac{\alpha}{10 \sqrt{-R} \beta}, k_{1}=k_{2}=b_{1}=b_{2}=0, a_{2}=\frac{3 \alpha^{2}}{25 R \beta}, \\
a_{0}=\frac{2500 \beta^{3} R \gamma F_{1}(t)^{2}+3 \alpha^{4}-25 \alpha^{2} \beta}{25 \alpha^{2} \beta}, \\
a_{1}=\frac{6 \alpha^{2}}{25 \sqrt{-R} \beta}, q=F_{1}(t) y+F_{2}(t) .
\end{gathered}
$$

Case 5.

$$
\begin{gathered}
a_{1}=-\frac{3 \alpha^{2}}{25 \sqrt{-R} \beta}, k_{1}=\frac{3 \alpha^{2} R}{25 \sqrt{-R} \beta}, q=F_{1}(t) y+F_{2}(t), \\
a_{2}=\frac{3 \alpha^{2}}{100 R \beta}, b_{1}=b_{2}=0, k_{2}=\frac{3 R \alpha^{2}}{100 \beta}, p=\frac{\alpha}{20 \sqrt{-R} \beta}, \\
a_{0}=\frac{20000 \beta^{3} R \gamma F_{1}(t)^{2}+3 \alpha^{4}-50 \alpha^{2} \beta}{50 \alpha^{2} \beta}
\end{gathered}
$$


Case 6.

$$
\begin{gathered}
a_{1}=\frac{3 \alpha^{2}}{25 \sqrt{-R} \beta}, k_{1}=-\frac{3 \alpha^{2}}{25 \sqrt{-R} \beta}, a_{2}=\frac{3 \alpha^{2}}{100 R \beta}, \\
b_{1}=b_{2}=0, k_{2}=\frac{3 R \alpha^{2}}{100 \beta}, \\
a_{0}=\frac{20000 \beta^{3} R \gamma F_{1}(t)^{2}+3 \alpha^{4}-50 \alpha^{2} \beta}{50 \alpha^{2} \beta}, \\
p=-\frac{\alpha}{20 \sqrt{-R} \beta}, q=F_{1}(t) y+F_{2}(t) .
\end{gathered}
$$

Case 7.

$$
\begin{gathered}
q=F_{1}(t) y+F_{2}(t), k_{1}=k_{2}=0, a_{2}=\frac{6 \alpha^{2}}{25 R \beta}, \\
b_{2}=-\frac{6 \alpha^{2}}{25 R \beta}, a_{1}=-\frac{6 \alpha^{2}}{25 \sqrt{-R} \beta}, \\
a_{0}=\frac{625 \beta^{3} R \gamma F_{1}(t)^{2}+6 \alpha^{4}-25 \alpha^{2} \beta}{25 \alpha^{2} \beta} \\
p=\frac{\alpha}{5 \sqrt{-R} \beta}, b_{1}=\frac{6 \alpha^{2}}{25 \sqrt{-R} \beta}
\end{gathered}
$$

Case 8.

$$
\begin{gathered}
b_{2}=\frac{6 \alpha^{2}}{25 R \beta}, q=F_{1}(t) y+F_{2}(t), k_{1}=k_{2}=0, \\
a_{1}=\frac{6 \alpha^{2}}{25 \sqrt{-R} \beta}, a_{2}=\frac{6 \alpha^{2}}{25 R \beta} \\
a_{0}=\frac{625 \beta^{3} R \gamma F_{1}(t)^{2}+6 \alpha^{4}-25 \alpha^{2} \beta}{25 \alpha^{2} \beta} \\
p=-\frac{\alpha}{5 \sqrt{-R} \beta}, b_{1}=\frac{6 \alpha^{2}}{25 \sqrt{-R} \beta} .
\end{gathered}
$$

Case 9.

$$
\begin{gathered}
b_{2}=\frac{6 \alpha^{2}}{25 R \beta}, q=F_{1}(t) y+F_{2}(t), k_{1}=k_{2}=0, \\
a_{1}=-\frac{6 \alpha^{2}}{25 \sqrt{-R} \beta}, a_{2}=\frac{6 \alpha^{2}}{25 R \beta}, \\
a_{0}=\frac{625 \beta^{3} R \gamma F_{1}(t)^{2}+6 \alpha^{4}-25 \alpha^{2} \beta}{25 \alpha^{2} \beta}, \\
p=\frac{\alpha}{5 \sqrt{-R} \beta}, b_{1}=-\frac{6 \alpha^{2}}{25 \sqrt{-R} \beta} .
\end{gathered}
$$

Case 10.

$$
\begin{aligned}
& a_{0}=\frac{625 \beta^{3} R \gamma F_{1}(t)^{2}+6 \alpha^{4}-25 \alpha^{2} \beta}{25 \alpha^{2} \beta}, k_{1}=k_{2}=0, \\
& a_{2}=\frac{6 \alpha^{2}}{25 R \beta}, a_{1}=\frac{6 \alpha^{2}}{25 \sqrt{-R} \beta}, b_{2}=-\frac{6 \alpha^{2}}{25 R \beta}, \\
& q=F_{1}(t) y+F_{2}(t), p=-\frac{\alpha}{5 \sqrt{-R} \beta}, b_{1}=-\frac{6 \alpha^{2}}{25 \sqrt{-R} \beta} .
\end{aligned}
$$

From (3.38b), (2.4) and Case $1-$ Case 10, we can obtain the following three types of solutions for the two-dimensional KdV-Burgers equation. Here we only consider the tanh-type solutions.

Type 1: From Case 1-Case 4, we obtain the solutions

$u_{1}=a_{0}+\frac{6 \alpha^{2}}{25 \beta} \tanh (\sqrt{-R} \xi)+\frac{3 \alpha^{2}}{25 \beta} \tanh ^{2}(\sqrt{-R} \xi)$

where $R<0, a_{0}=\left(2500 \beta^{3} R \gamma F_{1}(t)^{2}+3 \alpha^{4}-\right.$ $\left.25 \alpha^{2} \beta\right) / 25 \alpha^{2} \beta, \xi=-\alpha x / 10 \sqrt{-R} \beta+F_{1}(t) y+F_{2}(t)$.

Type 2: From Case 5 - Case 6 , we obtain solutions

$$
\begin{aligned}
u_{2}= & a_{0}+\frac{3 \alpha^{2}}{25 \beta}[\tanh (\sqrt{-R} \xi)+\operatorname{coth}(\sqrt{-R} \xi)] \\
& +\frac{3 \alpha^{2}}{100 \beta}\left[\tanh ^{2}(\sqrt{-R} \xi)+\operatorname{coth}^{2}(\sqrt{-R} \xi)\right],
\end{aligned}
$$

where $R<0, a_{0}=\left(20000 \beta^{3} R \gamma F_{1}(t)^{2}+3 \alpha^{4}-\right.$ $\left.50 \alpha^{2} \beta\right) / 50 \alpha^{2} \beta, \xi=-\alpha x / 20 \sqrt{-R} \beta+F_{1}(t) y+F_{2}(t)$.

Type 3: From Case 7 -Case 10, we obtain solutions

$$
\begin{aligned}
u_{31}= & a_{0} \pm \frac{6 \alpha^{2}}{25 \beta} \tanh (\sqrt{-R} \xi)+\frac{6 \alpha^{2}}{25 \beta} \tanh ^{2}(\sqrt{-R} \xi) \\
& \pm \mathrm{i} \frac{6 \alpha^{2}}{25 \beta} \operatorname{sech}(\sqrt{-R} \xi)[1 \pm \tanh (\sqrt{-R} \xi)],
\end{aligned}
$$

where $a_{0}=\left(625 \beta^{3} R \gamma F_{1}(t)^{2}+6 \alpha^{4}-25 \alpha^{2} \beta\right) / 25 \alpha^{2} \beta$, $\xi=-\alpha x / 5 \sqrt{-R} \beta+F_{1}(t) y+F_{2}(t)$.

\section{Remark 4:}

1. When setting the arbitrary functions $F_{1}(t)=-d \alpha$ $/ 10 \sqrt{-R} \beta$ and $F_{2}(t)=-c \alpha / 10 \sqrt{-R} \beta$, solution (3.49) is the same as the solutions obtained in $[8,9$, $13,17,25,26]$.

2. When setting the arbitrary functions $F_{1}(t)=-d \alpha$ $/ 20 \sqrt{-R} \beta$ and $F_{2}(t)=-c \alpha / 20 \sqrt{-R} \beta$, the solutions obtained in [17] can be recovered by (3.50). 


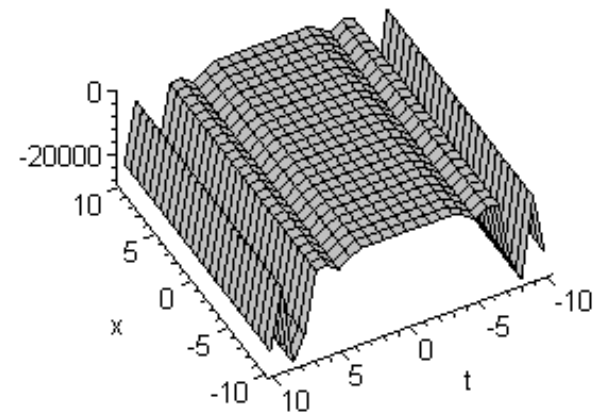

Fig. 1. The solution (3.49), where $R=-0.1, \alpha=2, \beta=$ $y=1, \gamma=3, F_{1}(t)=t^{2} \sin t, F_{2}(t)=\cos t$.

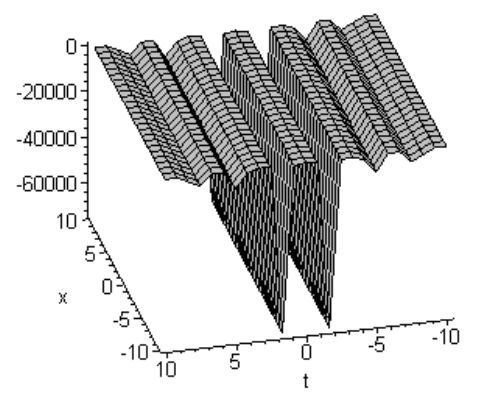

Fig. 2. The solution (3.49), where $R=-1, \alpha=-2, \beta=3$, $y=1, \gamma=3, F_{2}(t)=t \tan t, F_{2}(t)=t^{4}$.

3. When setting $F_{1}(t)=-d \alpha / 5 \sqrt{-R} \beta$ and $F_{2}(t)=$ $-c \alpha / 5 \sqrt{-R} \beta$, solution (3.51) includes the solution obtained in [13].

4. Using the generalized hyperbolic-function method [18, 19], we only obtained the solutions (3.49) and (3.51), but we did not obtain other formal solutions.

As illustrative samples, some properties of the solutions (3.49) - (3.50) are shown by Figures $1-4$.

\section{Conclusions}

Based on computerized symbolic computation, by introducing a new, more general ansatz than the ansatz in the extended tanh-function method, the modified extended tanh-function method, and the generalized hyperbolic-function method, we propose a generalized Riccati equation expansion method for searching for exact soliton-like solutions of nonlinear partial differential equations (PDEs), implemented by computer symbolic systems. Making use of our method and

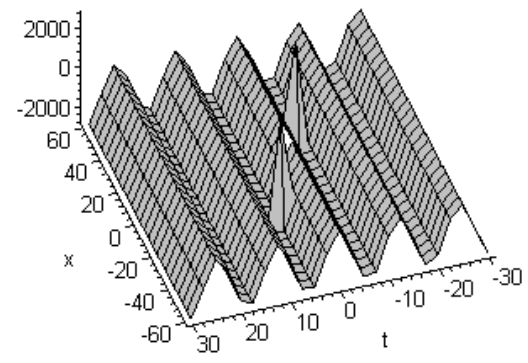

Fig. 3. The solution (3.50), where $R=-1, \alpha=3, \beta=4$, $y=6, \gamma=2, F_{2}(t)=t \sin t, F_{2}(t)=t$.

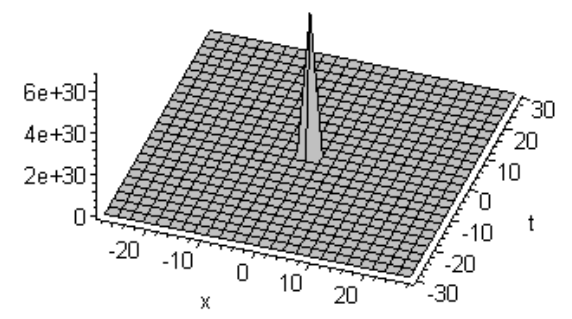

Fig. 4. The solution (3.50), $R=-1, \alpha=3, \beta=4, y=6$, $\gamma=2, F_{2}(t)=t, F_{2}(t)=t$.

maple, we studied the $\mathrm{cKdV}$ equation, the PKP equation, and the two-dimensional KdV-Burgers equation and obtained new families of exact solutions. In our exact solutions, the restriction on $\xi$ to be a linear function $x, y, t$ is removed and, with no extra effect, singular soliton-like solution and periodic solutions are obtained. It is shown that our method can not only deal with constant coefficients of nonlinear evolution equations (NEEs), but also with variable coefficients. To make the work feasible, how to choose the forms of $a_{0}, a_{i}, b_{i}, k_{i}(i=1, \cdots, m)$ and $\xi$ in the ansatz is the key step of our method. The method, proposed in this paper for one equation, may be extended to find exact solutions of other NEEs and coupled NEEs.

\section{Acknowledgements}

The authors would like to express their sincere thanks to the referees and editors of ZNA for their suggestions and help. The work is supported by the 
National Natural Science Foundation of China under the Grant No. 1007201, the National Key Basic Re-

[1] C. S. Gardner, J. M. Green, M. D. Kruskal, and R.M. Miura, Phys. Rev. Lett. 19 (1967) 1095.

[2] M. J. Ablowitz and P. A. Clarkson, Soliton, Nonlinear Evolution Equations and Inverse Scattering. Cambridge University Press, New York 1991.

[3] C. H. Gu, Soliton Theory and its Application, Zhejiang Science and Technology Press, Zhejiang 1990.

[4] R. Hirota, Phys. Rev. Lett. 27, 1192 (1971).

[5] W. P. Hong and Y.D. Jung, Z. Naturforsch. 54a, 549 (1999).

[6] E. J. Parkes, B. R. Duffy, Comput. Phys. Commun. 98, 288 (1996).

[7] E. J. Parkes and B. R. Duffy, Phys. Lett. A 229, 217 (1997).

[8] Z. B. Li and M.L. Wang, J. Phys. A: Math. Gen. 26, 6027 (1993).

[9] E. J. Parkes, J. Phys. A: Math. Gen. 27, L497 (1994).

[10] A.H. Khater, W. Malfiet, D. K. Callebaut, and E.S. Kamel, Chaos, Solitons, and Fractals 14, 513 (2002).

[11] E. Fan, Phys. Lett. A 277, 212 (2000).

[12] E. Fan, Z. Naturforsch. 56a, 312 (2001).

[13] E. Fan, J. Zhang, and Y. C. Benny, Phys. Lett. A 291, 376 (2001). search Development Project Program under the Grant No. G1998030600.

[14] Z. Y. Yan, Phys. Lett. A 292, 100 (2001).

[15] Z. Y. Yan, Z. Naturforsch. 56a, 809 (2002).

[16] Z. Y. Yan, H. Q. Zhang, Phys. Lett. A 285, 355 (2001).

[17] S. A. Elwakil, S. K. El-labany, M. A. Zahran, and R. Sabry, Phys. Lett. A 299, 179 (2002).

[18] Y. T. Gao and B. Tian, Comput. Phys. Commun. 133, 158 (2001).

[19] B. Tian and Y. T. Gao, Z. Naturforsch. 57a, 39 (2002).

[20] Y. Chen, B. Li, and H. Q. Zhang, Commun. Theor. Phys. (Beijing, China) 38, 261 (2002).

[21] B. Li, Y. Chen, and H. Q. Zhang, J. Phys. A: Math. Gen. 35, 8253 (2002).

[22] B. Li, Y. Chen, and H. Q. Zhang, Chaos, Solitons, and Fractals 15, 647 (2003).

[23] B. Li, Y. Chen, and H. Q. Zhang, Z. Naturforsch. 57a, 874 (2002).

[24] M. Senthilvelan, Appl. Math. Comput. 123, 381 (2001).

[25] W. X. Ma, J. Phys. A: Math. Gan. 26, L17 (1993).

[26] M. L. Wang, Y. B. Zhou, and Z. B. Li, Phys. Lett. A 216, 67 (1996). 